\title{
Research on the Integrated Framework of Modeling and Simulation on Equipment Deployment of the Chemical Reconnaissance System
}

\author{
Guohui Yan ${ }^{1, a}$, Xuezheng Zhu ${ }^{2, b}$, Jin Gu ${ }^{3, c}$, Kunlin Nie ${ }^{4, d}$, Wenjie Zhu ${ }^{5, e}$ \\ Institute of NBC Defence, Beijing, 102205, China \\ aygh7678@sina.com
}

Keywords: Chemical Reconnaissance; Equipment Deployment; Modeling and Simulation

\begin{abstract}
Aiming at the issues about equipment deployment of chemical reconnaissance in systemic level, the modeling thinking of chemical reconnaissance system is studied based on the ability theory, the simulation and calculation for the equipment application of the specific action-oriented chemical reconnaissance is discussed based on the Voronoi diagram theory, on this basis, the integrated framework of modeling and simulation is put forward, and finally, the validity of the framework is verified by some living examples.
\end{abstract}

\section{Introduction}

With the appearance of the information technology equipment and long-range precision striking weapons, the application issue about the overall equipment operation, marked with "systemic confrontation", have gradually infiltrated into the combat theory, campaign planning and other aspects of operations, which is playing an increasingly important role on the end and process of the war. ${ }^{[1]}$ Under this background, the research on equipment application has become a new research hotspot of all services and arms and the field of joint operations. In the case of the limited performance and scale of chemical reconnaissance equipment, how to optimize the combination and application method of multi-class, multi-type and different-function chemical reconnaissance equipment, explore effective measures to enhance the ability of chemical reconnaissance equipment system, has an important practical significance for improving the chemical protection ability and the chemical emergency handling ability of our army.

At present, the application methods of chemical reconnaissance equipment in training exercises are mostly summed up through the repeated exercises of their respective units, the empirical component is higher. Faced with the specific tasks, choosing which equipment, how much the amount and how to match with each other, those are all difficult questions, which have a great impact on the chemical protection and chemical emergency action. If there are relatively quantitative decision-making assisted models or tools, which will greatly reduce the difficulty of decision-making, improve the efficiency of decision-making and fully exert the overall efficiency of all the equipment and facilities in the chemical reconnaissance system.

\section{An Overview on Equipment Deployment of the Chemical Reconnaissance System}

The main task of chemical reconnaissance is to discover the symptoms of chemical attacks, identify the types of toxic agents, the infected cases and the secondary chemical hazards within the area of operations, as well as monitoring the spread and retention of the toxic agents clouds ${ }^{[2]}$, which provides the comprehensive and reliable chemical information for commanders to make decisions, and it's of great importance to guide chemical protection, exclude false alarms, eliminate chemical concerns, ensure the emotional stability of the troops, and make the operation intentions and actions without interference, so that it plays a very important role in the joint operations. In information operations, the professional equipment for chemical defense, the three-defense equipment of synthetic forces and the chemical reconnaissance equipment in main battle equipment need to be unified to set up a chemical reconnaissance system, so as to form the systematic chemical reconnaissance ability. In the system, all the equipment or devices involved in chemical reconnaissance are referred to as chemical reconnaissance nodes hereinafter. The limited chemical 
reconnaissance nodes should be used to ensure multiple important goals, whether the application is reasonable or not will directly affect the success rate of the chemical protection tasks.

The core of the issues about equipment application of the chemical reconnaissance system is the optimization of the system capability, the analysis for system capability should be carried out on the basis of the description of the chemical reconnaissance equipment system which is faced with the specific operation tasks, so as to achieve the goal of optimizing the overall application. While at present, the purpose of most research on system structure is to demonstrate the development demand of weaponry, the research on system structure has not been combined with the research of equipment application. Combining the ability theory with the Voronoi diagram theory, carrying out the research on model building and simulation experiment for equipment application of the chemical reconnaissance in systemic level, then putting forward the framework of modeling and simulation on equipment application of the chemical reconnaissance system, which provide a practical and feasible research route for the application of chemical reconnaissance equipment, and offer a certain basis for conducting the research on the deployment of chemical defense equipment.

Chemical reconnaissance ability refers to the summation of the abilities of all the equipment and devices in the chemical reconnaissance equipment system carrying out the collaborative observation, report, chemical agent detection, monitoring and test for the chemical weapon attacks and secondary chemical hazards. It includes the static reconnaissance ability and the dynamic reconnaissance ability, hereinto, the static reconnaissance is the observation and report for chemical hazards in this range when the position of each reconnaissance node in the chemical reconnaissance equipment system is relatively fixed, the dynamic reconnaissance refers to the abilities that the reconnaissance nodes in the chemical reconnaissance equipment system detect, monitor and test the toxicant infected cases of regions, roads and important goals in the event of enemy chemical weapon attacks or secondary chemical hazards.

\section{Framework Design of the Modeling and Simulation}

Modeling and simulation on the equipment application of chemical reconnaissance system is guided by the ability theory and Voronoi diagram theory, based on the establishment of the chemical reconnaissance task model and the equipment capacity model of chemical reconnaissance system, the stage division is implemented according to the chemical reconnaissance tasks, then the research on the issues about the demand calculation of chemical reconnaissance nodes, the optimization of the static reconnaissance allocation and the dynamic reconnaissance allocation is carried out. The design of the overall framework is shown in Figure 1.

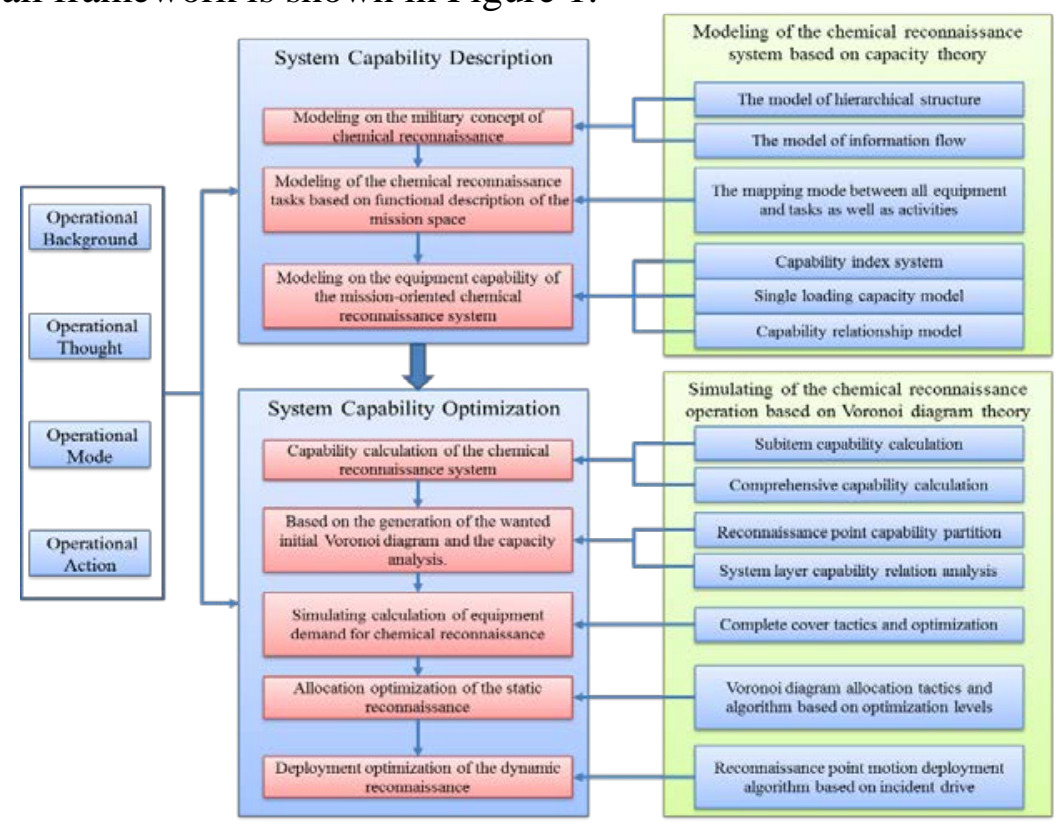

Figure 1. Framework of modeling and Simulation on equipment Deployment of the chemical reconnaissance system 
Faced with the specific tasks, the general idea of the equipment application of chemical reconnaissance system is to carry out an overall optimization for the components, the composition elements and the allocations of chemical reconnaissance system and make the overall ability of the system hold the advantage under the constraints of the existing equipment and operational conditions. ${ }^{[3]}$ The process of modeling and simulation includes the two main parts: system capability description, system capability optimization.

(1) Modeling on the military concept of chemical reconnaissance. The task is to qualitatively analyze the operation background, operation thought, operation pattern, operation action and other high-level and macroscopical operation cognitive problems of the chemical reconnaissance system, confirm the level and information flow of that, and determine a military background which is roughly consistent with the objective reality for the simulation calculation of chemical reconnaissance system.

(2) Modeling of the chemical reconnaissance tasks based on functional description of the mission space. By using the ideas of functional description for mission space, the chemical reconnaissance tasks are analyzed, setting up a standardized description for the correlativity and interaction between the process, entity, environment and other factors in the chemical reconnaissance tasks and the specific mission, task and action, establishing the mapping relationship between all equipment and tasks as well as activities in the chemical reconnaissance system, which provides the basis for capability modeling of the chemical reconnaissance system.

(3) Modeling on the equipment capability of the mission-oriented chemical reconnaissance system. The synergic relationship between the demand situation of implementing the chemical reconnaissance tasks for the equipment capacity of chemical reconnaissance system and the ability is revealed by modeling, constructing the mapping matrix about the operation task, capability and equipment of chemical reconnaissance system, setting up the concept lattice of equipment - ability operation task ${ }^{[4]}$, and then extracting the incidence relations among the abilities of the mission-oriented chemical reconnaissance system to establish the corresponding algorithm, which provides a guidance for the calculation and optimization of system capability.

(4) Capability calculation of the chemical reconnaissance system. According to the capability index model, single loading capacity model and capability relationship model established in previous step, the subentry ability and comprehensive ability of all kinds of equipment are calculated. The data of the point set and weight are provided for generating the weighted Voronoi diagram about the ability of the chemical reconnaissance system.

(5) Based on the generation of the wanted initial Voronoi diagram and the capacity analysis. According to the initial allocation system of the wanted chemical reconnaissance forces, the allocation data of each chemical reconnaissance node is extracted as the generating element, monitoring the warning ability, accusation ability, poison detecting ability, maneuver ability, testing ability and comprehensive ability respectively, setting the ability range of various types of equipment as the weight, then the weighted Voronoi diagram in the initial allocation of the chemical reconnaissance system is generated in Arcgis respectively, which provides the basis for the demand calculation and allocation optimization of chemical reconnaissance equipment ${ }^{[5]}$.

(6) Simulating calculation of equipment demand for chemical reconnaissance. According to the characteristics of the Voronoi diagram, which includes good proximity, contiguity, maximum circle, quickly divide the region as well as add and delete nodes, distinguishing the first, the second and the third kind of chemical reconnaissance nodes, firstly, positioning the coverage leak areas in the initial allocation system, then adding up and recording the vertex information of Voronoi diagram, as well as putting the vertex of the uncovered Voronoi diagram into the allocation points(covering the hole area) to improve the coverage rate of the protection area, repeating the operation until realizing the complete cover ${ }^{[6]}$.

(7) Allocation optimization of the static reconnaissance. Based on the Voronoi diagram and the maximum hollow circle strategy, the simulating calculation ranks the configurable positions of the third kind of nodes in a descending order under the condition of the limited equipment and the allocation for the first and second kind of nodes is completed, combing with protecting the priority 
of important targets, to study the optimization algorithm about allocation, and determine the allocation positions of the new adding chemical reconnaissance nodes; then researching on the allocation adaptation model of the third kind of nodes based on the field theory, and optimizing the allocation positions of the third kind of nodes with limited quantities.

(8) Deployment optimization of the dynamic reconnaissance. After encountering the chemical weapons attack or secondary chemical hazards, the chemical reconnaissance is aiming at the event area with priority, combing with the Voronoi diagram and virtual force thought, the mathematical model is established, and then carrying out the research on the optimal deployment issues of the third and fourth kind of chemical reconnaissance nodes.

\section{Instances of the model and simulation}

Based on the above framework, the simulating optimization for the equipment deployment of chemical reconnaissance system used to protect the offensive operations of a certain region is carried out. Due to limited space, the main process model and simulation results are given below. Firstly, the mapping matrix of the operational tasks, capability and equipment of chemical reconnaissance system is established according to the mission and task analysis, which is shown in figure 2; and then extracting the area of operation region, equipment type, the quantity and other parameters from the combat scenario, calculating the subentry ability and comprehensive ability according to the ability model, and the simulation analysis is carried out based on the generated Voronoi diagram, based on the situation of capacity analysis, the priority area is determined combined with the distribution status of the protection targets; finally, the simulating optimization allocation is carried out under the condition of the limited equipment, the weighted Voronoi diagram in Figure 3 clearly and directly demonstrates the capability distribution after allocation, which can well meet the needs of chemical reconnaissance of the front position and the important protection targets(blue square area). Once encountering the chemical weapons attack or secondary chemical hazards, the dynamic deployment optimization aiming at the event areas can be carried out on this basis.

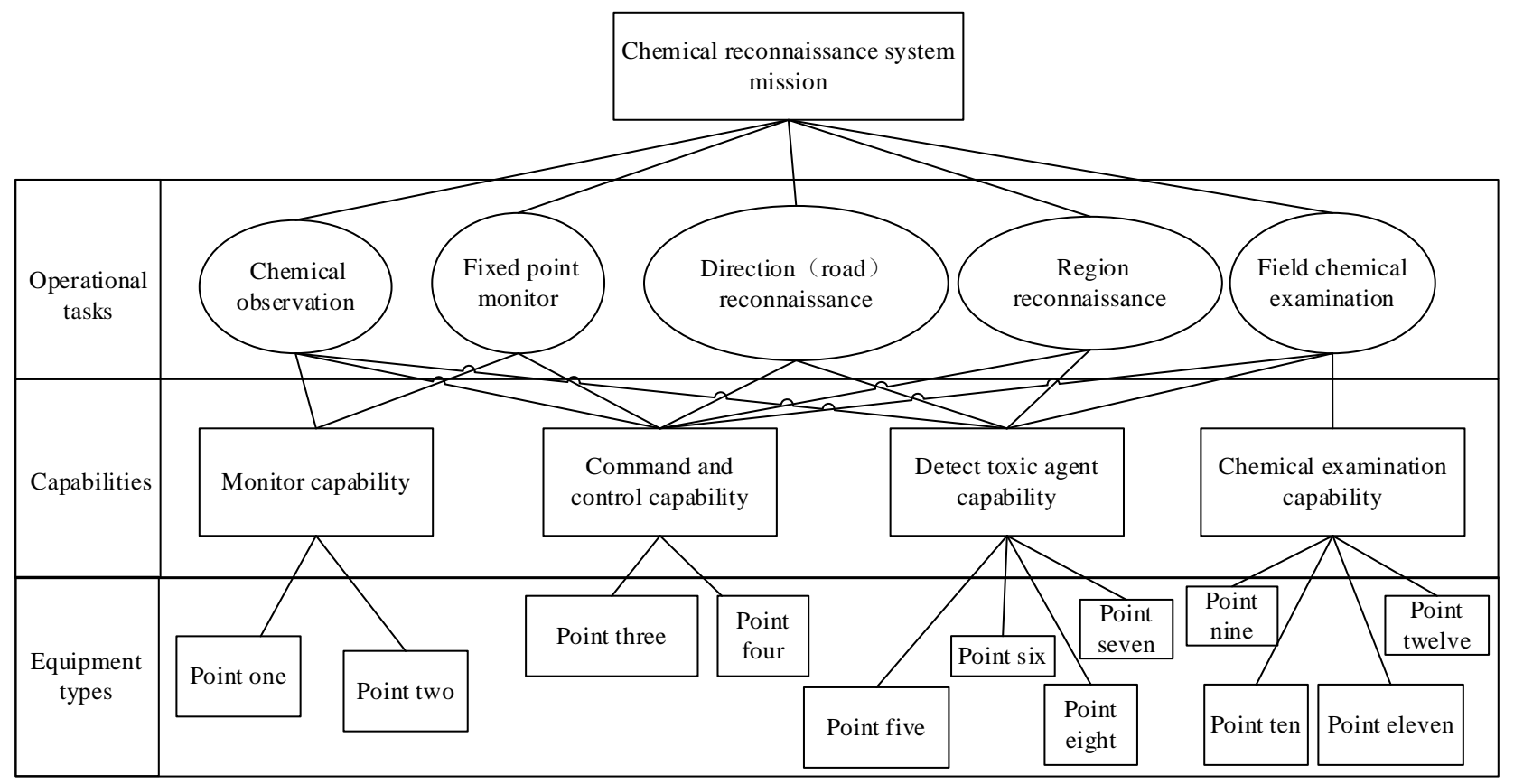

Figure 2. The mapping relation among operational task, capability and equipment of the chemical reconnaissance system 


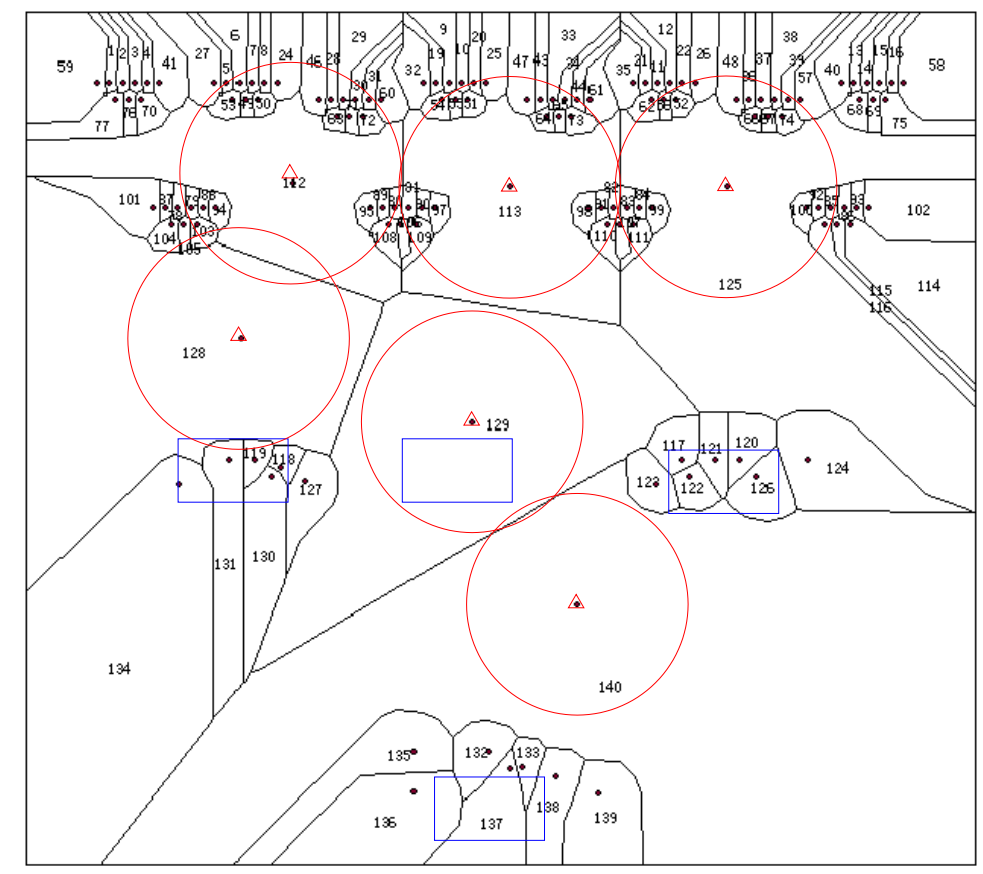

Figure 3. The weighted Voronoi diagram of the optimized allocation for chemical reconnaissance equipment

\section{Conclusion}

From the living examples of the model and simulation we can see, the framework of modeling and simulation, based on equipment application of the chemical reconnaissance system, can display the distribution situation about the equipment capability of the battlefield for commanders and optimize the deployment of computing-assisted functions, which will greatly improve the scientificity and efficiency of the command and decision-making. But the ability model given by the instances is still not so forceful, the allocation and optimization deployment have not been combined with the topography, geomorphology, meteorology and other environmental factors. Combing with the geographic information, the focus of the next research step is to establish the display model of on-site situation and set up the allocation and deployment algorithm under the condition of obstacles.

\section{Reference}

[1] Wanmin Cheng. Research on the Operational Application Issues of Air Force Weapons and Equipment [J]. Journal of National Defense University, 2005,5:86-88.

[2] Military Terminology Management Committee, Academy of Military Sciences. Military Terminology of the Chinese People's Liberation Army[S].Beijing: Military Science Press,2011:842

[3] Wanyong Cai etc. A New Optimization Model for the Operational Capability of the Warning and Monitoring Equipment System of Air Defense Radar [J]. Systems Engineering and Electronics,2010,32(10):2186-2190

[4] Guoqing Ye. Modeling on the architecture of weapons and equipment based on the mission and method framework[J]. Fire Control \& Command Control,2012,37(7):1-5

[5] S. Garrido, L. Moreno and D. Blanco, Exploration of a cluttered environment using Voronoi Transform and Fast Marching[J], Robotics and Autonomous Systems, 2008,56(12):1069-1081

[6] Zefeng Qin. Research on the Voronoi coverage algorithm for wireless sensor networks based on the security monitoring[D].Taiyuan: Taiyuan University of Technology,2013:18 